\title{
Developing more precise structural descriptions of layered covalent organic frameworks using total scattering data
}

\author{
Maxwell W. Terban $^{1}$, Lars Grunenberg ${ }^{1,2}$, Alexander M. Pütz ${ }^{1,2}$, Sebastian Bette ${ }^{1}$, Gökcen Savasci ${ }^{1,2,3}$, \\ Robert E. Dinnebier ${ }^{1}$, Bettina V. Lotsch ${ }^{1,2,3}$ \\ ${ }^{I}$ Max Planck Institute for Solid State Research, Stuttgart, Germany; \\ ${ }^{2}$ Department of Chemistry, Ludwig-Maximilians-Universität (LMU), Munich, Germany; \\ ${ }^{3}$ Exzellenzcluster E-conversion, Garching, Germany and Center for NanoScience, Munich, Germany; \\ m.terban@fkf.mpg.de
}

Materials containing microporous networks are an important topic of study for the development of improved technologies for a wide variety of applications including catalysis and gas storage. Widespread interest in metal- and more recently covalent-organic frameworks (MOFs/COFs) endures due to wide-ranging topologies and functionalities imbued by apparently limitless combinations of structural building units. However, while the focus remains primarily on crystalline products, semantics of how to interpret said crystallinity can vary widely in different communities, sometimes leading to oversight of important aspects of the structure that have essential implications on the material properties, or help to understand formation and functionalization processes [1].

The characterization of layered COFs in particular is difficult due to the presence of only a few, low-angle peaks in their diffraction patterns. This has led to a longstanding, dichotomous relationship between expectations based on energetic calculations and the structures observed by diffraction. We have recently demonstrated an experimental resolution, by considering the total rather than just Bragg scattering, and pair distribution function (PDF) analysis, to show how high apparent symmetries can emerge from random, local offsets of the layers [2].

Equipped with a fresh tool-set to characterize these structures, this talk will discuss our on-going efforts in this area - leveraging different length-scale sensitivities of real- and reciprocal-space vantage points for building and fitting models to obtain a more precise depiction of the structural states contained within.

[1] Grunenberg, L., Savasci, G., Terban, M. W., Duppel, V., Moudrakovski, I., Etter, M., Dinnebier, R. E., Ochsenfeld, C. \& Lotsch, B. V. (2021). J. Am. Chem. Soc. 143, 3430.।

[2] Pütz, A. M., Terban, M. W., Bette, S., Haase, F., Dinnebier, R. E. \& Lotsch, B. V. (2020). Chem. Sci. 11, 12647.

Keywords: covalent organic frameworks (COFs), total scattering, pair distribution function (PDF) 